\title{
Quantum Poincaré Recurrences for Hydrogen Atom in a Microwave Field
}

\author{
Giuliano Benenti $^{(a)}$, Giulio Casati ${ }^{(b, c, d)}$, Giulio Maspero ${ }^{(e)}$, and Dima L. Shepelyansky ${ }^{(f)}$ \\ ${ }^{(a)}$ CEA, Service de Physique de l'Etat Condensé, Centre d'Etudes de Saclay, F-91191 Gif-sur-Yvette, France \\ ${ }^{(b)}$ International Center for the Study of Dynamical Systems, Via Lucini 3, \\ Università degli Studi dell'Insubria, 22100 Como, Italy \\ ${ }^{(c)}$ Istituto Nazionale di Fisica della Materia, Unità di Milano, Milano, Italy \\ ${ }^{(d)}$ Istituto Nazionale di Fisica Nucleare, Sezione di Milano, Milano, Italy \\ ${ }^{(e)}$ Università Pontificia della S. Croce, Piazza di S. Apollinare 49, 00186 Roma, Italy \\ ${ }^{(f)}$ Laboratoire de Physique Quantique, UMR 5626 du CNRS, Université Paul Sabatier, 31062 Toulouse, France
}

(October 1, 2018)

\begin{abstract}
We study the time dependence of the ionization probability of Rydberg atoms driven by a microwave field, both in classical and in quantum mechanics. The quantum survival probability follows the classical one up to the Heisenberg time and then decays algebraically as $P(t) \propto 1 / t$. This decay law derives from the exponentially long times required to escape from some region of the phase space, due to tunneling and localization effects. We also provide parameter values which should allow to observe such decay in laboratory experiments.
\end{abstract}

PACS: 32.80.Rm, 05.45.Mt

During the last two decades the manifestations of classical chaos in microwave ionization of Rydberg atoms have been studied experimentally by different groups and many interesting results have been obtained [1 4 . In particular, laboratory experiments showed the quantum suppression of classically diffusive ionization process, in agreement with the predictions of dynamical localization theory [5]. The experimental technique based on accelerated proton beams, which is used for production of hydrogen atoms, allows to obtain interaction times with the microwave field of only few hundreds microwave periods 1,2, 2. 4 . On the contrary, the thermal beams used with alkali Rydberg atoms allow to vary the interaction time by orders of magnitude up to $10^{5}$ microwave periods [3]. The first experiments of Walther's group [3] indicated an anomalously slow decay of the $10 \%$-ionization threshold field as a function of the interaction time. This result cannot be explained within the picture of diffusive ionization in the domain of classical chaos. Some suggestions have been put forward to explain this slow decay which was attributed to some possible effects of noise for such long interaction times [6.,7]. More recently, new experimental data for the behavior of the survival probability $P(t)$ with time have been presented [8], showing an algebraic law decay $P(t) \propto t^{-\alpha}$, with $\alpha \approx 0.5$. In the same paper, numerical simulations of quantum dynamics have been made, giving a value of $\alpha$ consistent with experimental data. The origin of the slow algebraic decay was attributed to the underlying structure of classical mixed phase space composed by integrable islands surrounded by chaotic components. However, the investigations of classical chaotic systems with mixed phase space showed that the probability of Poincaré recurrences to the same region, or the survival probability up to time $t$, decays algebraically with power $\alpha \approx 1.5-3[9-11$. Moreover, since the integral $\int_{t}^{\infty} P(\tau) d \tau$ is proportional to the measure of the finite chaotic region where the trajectory is trapped, the value of $\alpha$ should be greater than one. According to the correspondence principle, one expects that, in the semiclassical regime, classical and quantum systems exhibit the same decay law. Therefore the above exponent $\alpha \approx 0.5$ found in the experiments requires, in this respect, an explanation. In particular the question arises whether this obtained value is generic or corresponds to some initial transient time behavior in a regime where quantum effects play an important role.

Recent studies of quantum Poincaré recurrences for the Chirikov standard map in the semiclassical regime with mixed phase space [12] showed that quantum $P(t)$ follows the classical decay during a relatively large time $t_{H}$. The time $t_{H}$ gives the Heisenberg time scale, which is determined by inverse level spacings. For $t>t_{H}$, the quantum survival probability starts to decay inversely proportional to time $(\alpha=1)$ and becomes much larger than the classical one. The power $\alpha=1$ is due to exponentially long times required to escape from some region of phase space [12]. These exponentially long escape times are originated by tunneling from classically integrable region or by the exponential quantum localization. The above quantum behavior, with exponent $\alpha=1$, is different from the experimental data [8] and this constitutes an additional motivation for the present paper. Indeed the highly excited states of hydrogen atom in a microwave field can be described by the Kepler map which is very similar to the Chirikov standard map [5] and therefore one would expect the same behavior for the time dependence of survival probability.

In order to investigate the probability decay for the hydrogen atom in a microwave field, we choose the initial state with principal quantum number $n_{0}$ and numerically studied the survival probability $P(t)$ in a linearly polarized monochromatic electric field $\epsilon(t)=\epsilon \sin (\omega t)$. Here $\epsilon$ 
and $\omega$ are the strength and frequency of the microwave field, measured in atomic units. The quantum evolution is numerically simulated by the Kepler map [5], by the one-dimensional $(1 d)$ model of a hydrogen atom and by the $3 d$ model for atoms initially prepared in states extended along the field direction and with magnetic quantum number $m=0$. The comparison of these three models shows that the essential physics is captured by the $1 d$ model as already discussed in [5]. In addition we show that also the Kepler map gives an approximate correct description of the dynamics.

The hydrogen atom in a linearly polarized monochromatic electric field is described by the Hamiltonian

$$
H=\frac{p^{2}}{2}-\frac{1}{r}+\epsilon z \sin (\omega t),
$$

where, in the $1 d$ model the motion is assumed to take place along the field direction $(z$-axis, with $z \geq 0)$. In order to compare classical and quantum dynamics it is convenient to use the scaled field strength $\epsilon_{0}=\epsilon n_{0}^{4}$ and frequency $\omega_{0}=\omega n_{0}^{3}$, which completely determine the classical dynamics. The classical limit corresponds to $\hbar_{\mathrm{eff}}=\hbar / n_{0} \rightarrow 0$, at constant $\epsilon_{0}, \omega_{0}$. For $\omega_{0}>1$ the main change of the electron energy $E$ occurs when the electron is close to the nucleus. As a consequence the dynamics is approximately given by the Kepler map [5].

$$
\bar{N}=N+k \sin \phi, \quad \bar{\phi}=\phi+2 \pi \omega(-2 \omega \bar{N})^{-3 / 2},
$$

where $N=E / \omega, k=2.6 \epsilon \omega^{-5 / 3}, \phi=\omega t$ is the phase of the microwave field when the electron passes through the perihelion and the bar marks the new values of variables. In the quantum case, the change of $N$ gives the number of absorbed photons while the number of photons required to ionize the atom is $N_{I}=1 /\left(2 n_{0}^{2} \omega\right)$. In classical mechanics diffusive ionization takes place for fields above the chaos border: $\epsilon_{0}>\epsilon_{c} \approx 1 /\left(49 \omega_{0}^{1 / 3}\right)$ [5]. The quantum dynamics of the model (2) is described by the quantum Kepler map for the wave function $\psi(\phi)$ :

$$
\bar{\psi}=\exp \left(-i H_{0}(\hat{N})\right) \hat{P} \exp (-i k \cos \hat{\phi}) \psi
$$

where $H_{0}(\hat{N})=2 \pi / \sqrt{-2 \omega \hat{N}}, \hat{N}=-i d / d \phi, \hat{\phi}=\phi$ $(-\infty<\phi<+\infty)$, and the operator $\hat{P}$ projects probability over the states with negative energy $(N<0)$ [5]. We introduce an absorption border for levels with $n \geq n_{c}$, which for the Kepler map corresponds to $N \geq$ $N_{c} \approx-1 /\left(2 n_{c}^{2} \omega\right)$ 13]. Such border occurs in real laboratory experiments, for example as a consequence of unavoidable static electric field experienced by the Rydberg atoms during their interaction with the microwave field. The absorption border $n_{c}$ can be varied in a controlled way via a static electric field $\epsilon_{s}$, the static field ionization border being $\epsilon_{s} n_{c}^{4} \approx 0.13$.

The results of quantum simulations for the situation similar to the experimental one (Fig. 2 (b) in Ref. [8]) are shown in Fig. 1. The Kepler map description allows us to study the quantum dynamics up to very long times $\left(t=10^{8}\right.$, here and below time is given in microwave periods). In the case $n_{0}=23$ the quantum data for the survival probability $P(t)$ obtained from the quantum Kepler map and the $1 d$ hydrogen atom model agree with each other (see the inset of Fig. 11) and with the numerical computations of $\|8\|$. However all these data are strongly different from the classical probability decay shown in Fig.11, which displays a slope $\alpha \approx 2$. The reason of this disagreement should be attributed to the fact that $n_{0}=23$ is not in the semiclassical regime. Our data for the Husimi distribution, obtained from the Wigner function by smoothing over the size $\hbar$ [14], show that a significant part of the probability is trapped inside the stable island at $n \approx 20\left(\omega n^{3} \approx 1\right)$. For this reason the probability decays slowly during a long time $t \approx 10^{5}$ after which it drops faster. If $n_{0}$ is increased significantly, the semiclassical regime is reached and the quantum probability decay becomes close to the classical one up to the time scale $t_{H} \approx 10^{4}$. Our data show that $t_{H}$ is proportional to $n_{0}$ (at fixed $\epsilon_{0}, \omega_{0}$ ), in agreement with previous estimates of Ref. [12], according to which $t_{H} \propto 1 / \hbar_{\text {eff }}$. After this time the quantum $1 / t$ decay is clearly observed in agreement with the results of 12].

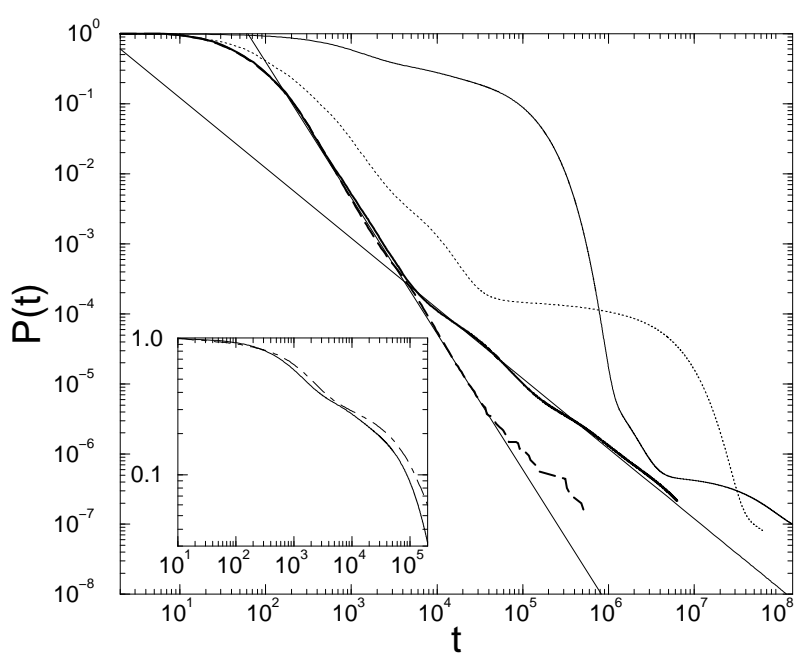

FIG. 1. Survival probability $P(t)$ as a function of the interaction time $t$ (in units of microwave periods) for $\epsilon_{0}=0.065$, $\omega_{0}=1.6, n_{c}=2 n_{0}$ : quantum Kepler map for $n_{0}=23$ (thin solid line), $n_{0}=230$ (thin dotted line), $n_{0}=2300$ (thick solid line) and classical Kepler map (thick dashed line, ensemble of $10^{9}$ trajectories). The straight lines have slopes 1 and 1.94, the latter coming from a fit of the classical decay for $2 \times 10^{2}<t<3 \times 10^{4}$. Inset: quantum Kepler map (solid line) versus $1 d$ hydrogen model (dot-dashed line) for $n_{0}=23$.

In Fig. 2 we show a more realistic case in which, initially, classical and quantum probabilities decay in a very similar way and where only after a time $t_{H} \approx 5 \times 10^{2}$, the quantum survival probability starts to decay more 
slowly $(P(t) \propto 1 / t)$ than the classical one which decays as $1 / t^{\alpha}$, with $\alpha \approx 2.15$. This case corresponds to $n_{0}=60$ and can be observed in experiments similar to those performed in [8]. Again the quantum Kepler map gives a qualitatively correct description of the ionization process up to very long interaction times. The comparison of quantum simulations for the $1 d$ hydrogen atom model and the $3 d$ dynamics is shown in the inset of Fig. 2. It demonstrates that both dynamics give very close results, confirming that the essential physics is captured by the $1 d$ model. We put the absorption border near the initial state $\left(n_{c}=64\right)$ in order to have $\rho_{c}=\ell_{\phi} / \Delta N_{c} \approx 3.5>1$, where $\ell_{\phi}=3.3 \epsilon_{0}^{2} \omega_{0}^{-10 / 3} n_{0}^{2}$ is the localization length in number of photons [5] and $\Delta N_{c}=\left(n_{0} / 2 \omega_{0}\right)\left(n_{0}^{2} / n_{c}^{2}-1\right)$ is the number of photons required to reach the absorption border. In this way the probability can go out very easily and the $1 / t$ probability decay is observed after a short transient time of the order of 20 microwave periods. On the contrary, when $\rho_{c}<1$, as in the case of Fig. 1 for $n_{0}=23\left(\rho_{c} \approx 0.3\right)$, strong fluctuactions around the $1 / t$ decay take place. This is analogous to the huge (log-normally distributed) conductance fluctuactions in a disorder solid with localization length smaller than the system size [15].

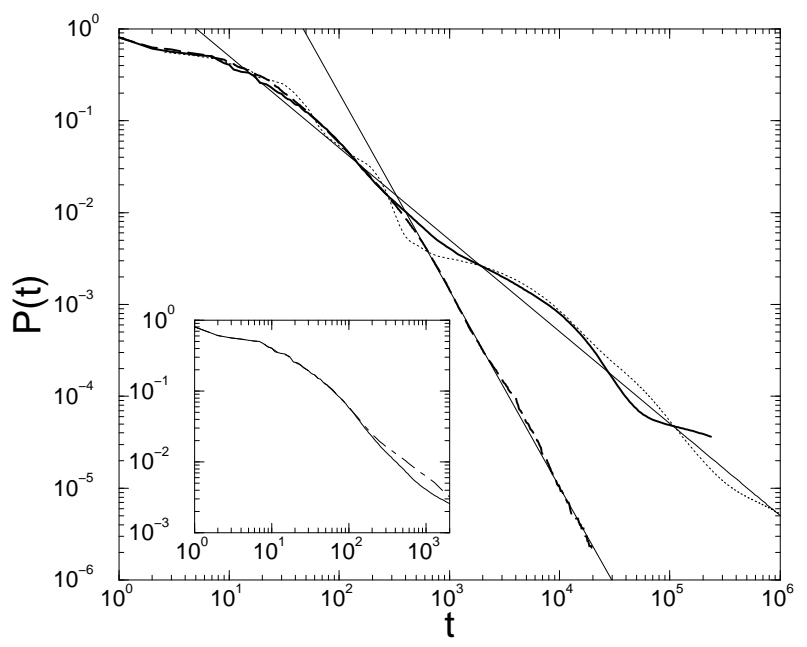

FIG. 2. Survival probability for $\epsilon_{0}=0.1, \omega_{0}=2.6$, $n_{0}=60, n_{c}=64$ : quantum Kepler map (dotted line), quantum (solid line) and classical (dashed line, ensemble of $3 \times 10^{6}$ trajectories) $1 d$ hydrogen model. The straight lines have slopes 1 and 2.15, the latter coming from a fit of the classical decay for $5 \times 10^{2}<t<2 \times 10^{4}$. Inset: quantum survival probability for the $1 d$ model (solid line) and the $3 d$ model (dot-dashed line).

In order to confirm that the algebraic probability decay is related to the sticking of classical trajectories and of quantum probability near the integrable islands in the phase space, we show in Fig. 3 the time evolution of the survival probability distribution in the phase space of action-angle variables $(n, \theta)$ for the $1 d$ model. In the classical case $3 \times 10^{6}$ orbits were initially homogenously distributed in the angle $\theta$ on the line $n=n_{0}=60$, corresponding to the initial quantum state with principal quantum number $n_{0}=60$. After 50 microwave periods, the classical distribution of non ionized orbits shows a fractal structure which surrounds the stability islands (Fig. 3 top left). At larger times this distribution approaches more and more closely the boundary critical invariant curves (Fig. 3 bottom left). One of them confines the motion in the region with $n>n_{b} \approx n_{0}\left(\epsilon_{c} / \epsilon_{0}\right)^{1 / 5} \approx 41$ where $n_{b}$ determines the classical chaos border for given $\epsilon_{0}$. Other invariant curves mark the critical boundaries around internal stability islands (for example at $n \approx 55$, corresponding to $\omega n^{3} \approx 2$ ). In the quantum case the value of $\hbar_{\text {eff }}$ is not sufficiently small to resolve the fractal structure at small scales. However, the Husimi function shows similarities with classical probability distribution at $t=50$ (Fig. 3 top right). At longer times, the diffusion towards the boundary at $n_{b}$ is slowed down due to localization effects and penetration of the quantum probability inside the classical integrable islands. At $t=10^{4}$ (Fig. 3 bottom right) the quantum probability is concentrated in a layer near $n_{b}$. Due to localization effects, the Husimi function does not change significantly for a very long interaction time $\left(10^{3}<t<3 \times 10^{4}\right)$. Eventually the probability starts to penetrate very slowly inside the main island at $n \approx n_{b}$. Therefore tunneling and localization effects are responsible for the slow $1 / t$ decay of the quantum survival probability seen in Fig. 2.

The fractal structure of the classical distribution is washed out at scales smaller than the minimal quantum cell $\hbar_{\text {eff }}$. Therefore a better resolution can be obtained increasing the principal quantum number $n_{0}$, at fixed $\epsilon_{0}$, $\omega_{0}$, and $n_{c} / n_{0}$. The Husimi function clearly reflects the underline fractal structure at very high principal quantum numbers $n_{0}=150$ (Fig. 团 left) and $n_{0}=1200$ (Fig. (1) right). Similar quantum fractals have been found in the kicked rotator model with absorbing boundary conditions [16].

Notice that the probability decay $P(t)$ is related to correlations decay via $C(t) \propto t P(t)$ [9]. In the case of $\alpha=1$ this implies that correlations do not decay. The Fourier transform of $C(t)$ gives the spectral density $S(\omega)$ of the effective noise produced by the dynamics: $S(\omega)=\int C(t) \exp (i \omega t) d t \approx 1 / \omega$. This shows that the spectral noise associated with the quantum Poincaré recurrences with $\alpha=1$ scales like $S(\omega) \propto 1 / \omega$. A similar behavior of noise has been observed in many scientific disciplines [17], for example in the resistence fluctuactions of different solid state devices 187 . This phenomenon is known as $1 / f$ noise and usually extends over several orders of magnitude in frequency, indicating a broad distribution of time scales in the system. In the case of quantum Poincaré recurrences this property stems from the exponentially low escape rate from some regions of the phase space. 
In summary, on the basis of our previous investigations and of the numerical studies presented in this paper we conclude that the survival probability for Rydberg atoms in a microwave field decays, up to the time scale $t_{H} \propto n_{0}$, in a way similar to the classical probability. For $t>t_{H}$ the quantum probability starts to decay slower than the classical one, with the exponent of the algebraic decay $\alpha=1$. We have given parameter values which should allow to observe quantum Poincaré recurrences in microwave experiments with Rydberg atoms.
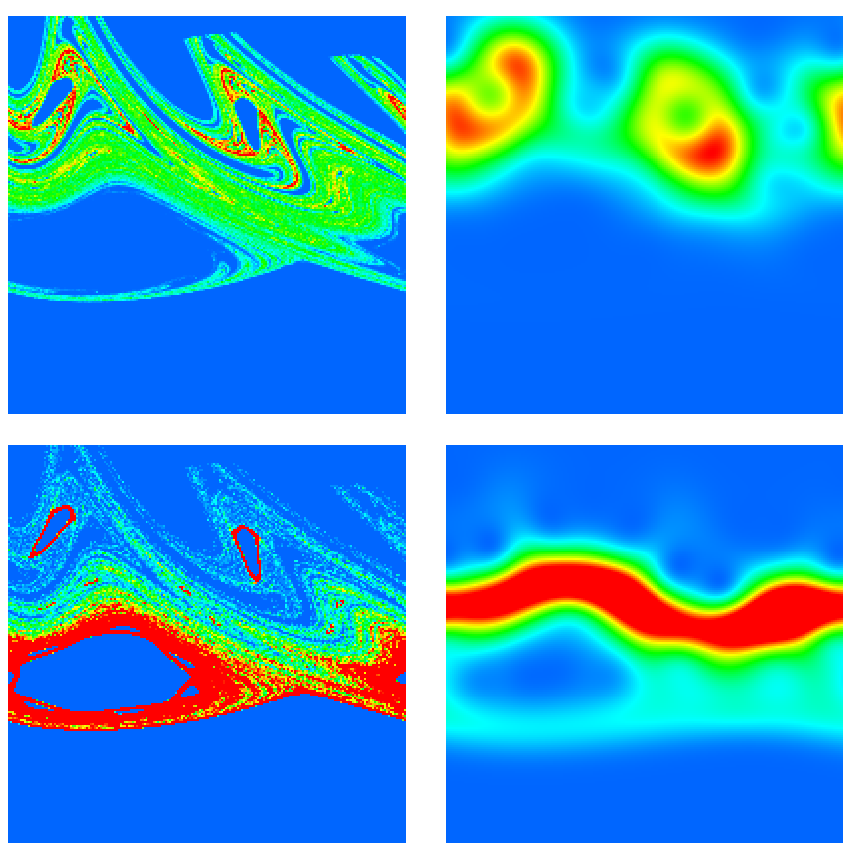

FIG. 3. Classical density plot (left) and Husimi function (right) in action-angle variables $(n, \theta)$, with $30 \leq n \leq 63.5$ (vertical axis) and $0 \leq \theta<2 \pi$ (horizontal axis), for the $1 d$ model in the case of Fig. 2. Husimi function is averaged in a finite time interval to decrease fluctuations: $50 \leq t \leq 60$ (top); $2 \times 10^{3} \leq t \leq 10^{4}$ (bottom left); $9.9 \times 10^{3} \leq t \leq 10^{4}$ (bottom right). The color is proportional to the density: blue for zero and red for maximal density.
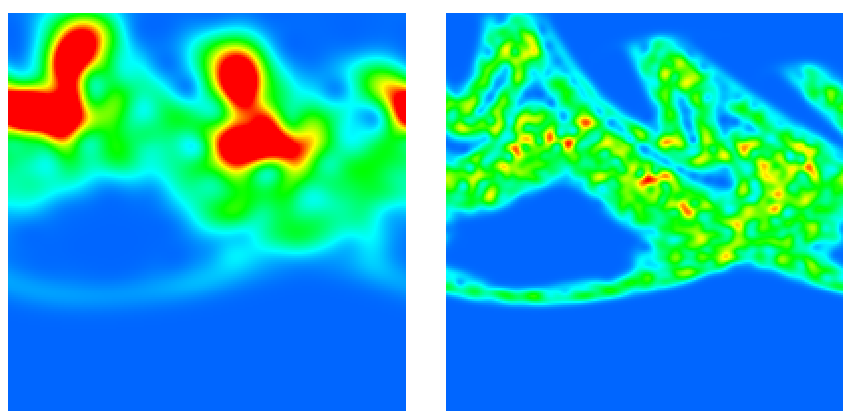

FIG. 4. Quantum fractal Husimi function for parameter values and interaction times as in Fig. 3 top left, with $n_{0}=150$ (left) and $n_{0}=1200$ (right).

This research is done in the frame of EC program RTN1-1999-00400.

[1] E.J. Galvez, B.E. Sauer, L. Moorman, P.M. Koch, and D. Richards, Phys. Rev. Lett. 61, 2011 (1988).

[2] J.E. Bayfield, G. Casati, I. Guarneri, and D.W. Sokol, Phys. Rev. Lett. 63, 364 (1989).

[3] M. Arndt, A. Buchleitner, R.N. Mantegna, and H. Walther, Phys Rev. Lett. 67, 2435 (1991).

[4] P.M. Koch and K.A.H. van Leeuwen, Phys. Rep. 255, 289 (1995).

[5] G. Casati, I. Guarneri, and D.L. Shepelyansky, IEEE J. Quant. Electron. 24, 1420 (1988).

[6] R. Blümel, A. Buchleitner, R. Graham, L. Sirko, U. Smilansky, and H. Walther, Phys. Rev. A 44, 4521 (1991).

[7] S. Fishman and D.L. Shepelyansky, Europhys. Lett. 16, 643 (1991).

[8] A. Buchleitner, D. Delande, J. Zakrzewski, R.N. Mantegna, M. Arndt, and H. Walther, Phys. Rev. Lett. 75, 3818 (1995).

[9] B.V. Chirikov and D.L. Shepelyansky, Physica D 13, 395 (1984); Phys. Rev. Lett. 82, 528 (1999).

[10] T. Geisel, A. Zacherl and G. Radons, Phys. Rev. Lett. 59, 2503 (1987).

[11] Y.-C. Lai, M. Ding, C. Grebogi and R. Blümel, Phys. Rev. A 45, 8284 (1992).

[12] G. Casati, G. Maspero, and D.L. Shepelyansky, Phys. Rev. Lett. 82, 524 (1999).

[13] We introduce complete absorption for levels with $n \geq n_{c}$ after each microwave period $(\Delta t=1)$. We checked that our results do not change significantly for a wide range of variation of $\Delta t$ around this value.

[14] We construct the Husimi function in a way similar to R.V. Jensen, M.M. Sanders, M. Saraceno, and B. Sundaram, Phys. Rev. Lett. 63, 2771 (1989).

[15] J.-L. Pichard, N. Zanon, Y. Imry, and A.D. Stone, J. Phys. France 51, 587 (1990).

[16] G. Casati, G. Maspero, and D.L. Shepelyansky, Physica D 131, 311 (1999).

[17] W.H. Press, Comments Astrophys. 7, 103 (1978).

[18] P. Dutta and P.M. Horn, Rev. Mod. Phys. 53, 497 (1981); M.B. Weissman, Rev. Mod. Phys. 60, 537 (1988). 